\title{
Production of Ansamycin Polyketide Precursors in Escherichia coli
}

\author{
M. A. Rude, C. Khosla \\ Dedicated to the memory of Professor Kenneth L. Rinehart Jr., for his pioneering studies \\ in natural product chemistry, including structure elucidation of the ansamycin antibiotic \\ geldanamycin.
}

Received: May 31, 2006 / Accepted: July 28, 2006

(C) Japan Antibiotics Research Association

\begin{abstract}
For the heterologous production of ansamycin polyketides such as rifamycin and geldanamycin in Escherichia coli, a number of unusual but important tools must be engineered into the bacterium. Here we demonstrate efficient production of the starter unit 3-amino-5-hydroxybenzoic acid (AHBA) and the methoxymalonyl extender unit in E. coli. Previous work has demonstrated the production of the ansamycin starter unit AHBA in E. coli in low yield. It was shown that the low yield is primarily due to acetylation of AHBA into $\mathrm{N}$ acetyl-AHBA. Three methods for minimizing this side reaction were evaluated. First, a putative $N$-arylamineacetyltransferase (NAT) was deleted from the E. coli chromosome, although this did not alter $N$-acetyl-AHBA production. Next, E. coli grown in media devoid of glucose yielded a nearly equal mixture of AHBA and $N$-acetylAHBA. Lastly, the NAT inhibitor glycyrrhizic acid was shown to further inhibit the acetylation reaction. The entire set of genes for synthesizing the methoxymalonyl extender unit was transferred from the geldanamycin producer Streptomyces hygroscopicus into E. coli. The pathway specific ACP isolated from the resulting recombinant strain was found to predominantly occur as methyoxymalonylACP. Together, these findings set the stage for engineered biosynthesis of ansamycin polyketides in E. coli.
\end{abstract}

C. Khosla (Corresponding author), M. A. Rude: Departments of Chemical Engineering, Chemistry, and Biochemistry, Stanford University, Stanford, California 94305, United States of America, E-mail:khosla@stanford.edu
Keywords polyketide, 3-amino-5-hydroxybenzoic acid, ansamycin, methoxymalonyl-ACP, Escherichia coli

\section{Introduction}

Polyketide natural products play an important role in the treatment of a variety of human diseases as well as in animal health and agriculture. Many natural producers of polyketides, typically bacteria and fungi, exhibit undesirable characteristics such as slow growth, low productivity, or poorly understood genetics. As a result, there is considerable interest in making these compounds in heterologous hosts such as Streptomyces coelicolor and Escherichia coli [1]. E. coli has gained attention as a possible heterologous host due to its well defined genetics, rapid growth characteristics, and the existence of highyielding secondary metabolite fermentation protocols. Previously, an E. coli system was engineered for the production of the polyketide 6-deoxyerythronolide B (6dEB) [2]. Biosynthesis of 6-dEB required synthesis of the non-native extender unit methylmalonyl-CoA in E. coli. Other classes of polyketides require unnatural starter and extender units such as 3-amino-5-hydroxybenzoic acid (AHBA) and methoxymalonyl-ACP, respectively. One class of polyketides that requires these polyketide building blocks is the ansamycins.

The ansamycins contain an aromatic ring bridged by a polyketide chain terminating in an amide linkage [3]. Some examples of ansamycins are the antibiotic rifamycin and the anti-tumor compounds geldanamycin and ansamitocin 


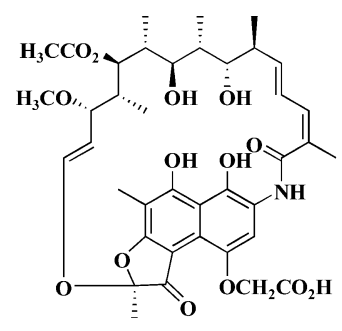

Rifamycin B

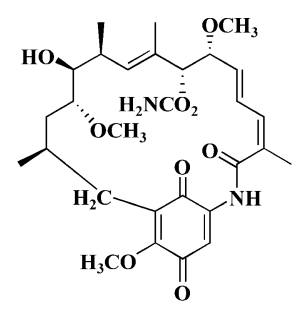

Geldanamycin

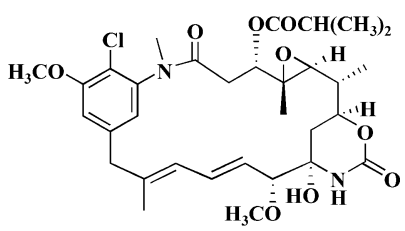

Ansamitocin P-3

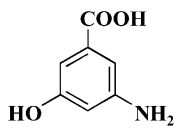

AHBA

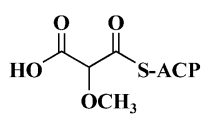

methoxymalonyl-ACP
Fig. 1 Structure of three ansamycin polyketides and the starter unit AHBA.

The extender unit methoxymalonyl-CoA is used to make ansamitocin P-3 and geldanamycin.

P-3 (Fig. 1). The biosynthesis of the ansamycins starts with the synthesis of AHBA, which is then loaded onto a type I polyketide synthase (PKS) where several rounds of elongation occur. The final polyketide chain is then cyclized to yield a macrocyclic lactam. MethylmalonylCoA and malonyl-CoA are used in chain elongation during the course of synthesis of all three aforementioned ansamycins [4 6]. In addition, geldanamycin and ansamitocin PKSs also use methoxymalonyl-ACP as an extender unit [7 9]. Thus, engineered biosynthesis of these ansamycins in $E$. coli would require that this heterologous host be able to produce these unusual building blocks.

Previous work has demonstrated low levels of AHBA production in E. coli [10] although there are no reports of methoxymalonyl-ACP production in this bacterium. In this work, we demonstrate that the expression of a hybrid rifamycin and ansamitocin AHBA gene cluster in E. coli leads to production of $N$-acetyl-AHBA under minimal media fermentation conditions with only low production of AHBA. The use of complex media, such as LB broth, results in a higher level of AHBA being produced. We also show that by expressing the $f k b G-K$ gene cluster from $S$. hygroscopicus in E. coli, methoxymalonyl-ACP could be generated. These two accomplishments create an E. coli platform that can be used for the production of ansamycin polyketides.

\section{Results and Discussion}

\section{AHBA Production}

Previously, a pET28 E. coli expression vector containing the rifH,N,M,K and asm23,47 genes (pKW255) produced $3 \mathrm{mg} /$ liter of isolated AHBA in minimal media with glucose as the sole carbon source [10]. To determine the cause of the low productivity, further analysis of the fermentation broth for side product formation was undertaken. $N$-Acetyl-AHBA was found to be the dominant product in these minimal media fermentations. From these fermentations, very little AHBA could be detected using HPLC analysis. To create a robust platform for making ansamycin polyketides in E. coli, methods for shifting the balance of $N$-acetyl-AHBA to AHBA are needed.

Three approaches were evaluated to inhibit the formation of $\mathrm{N}$-acetyl-AHBA. First, a putative $\mathrm{N}$-arylamine acetyl transferase (NAT), nhoA [11] was deleted from the E. coli genome based on the hypothesis that this enzyme was responsible for transferring an acetyl group from acetylCoA to AHBA. This gene was successfully deleted from the $E$. coli chromosome to generate MAR1. Shake flask fermentations of $E$. coli MAR1/pKW255 in minimal media revealed that $N$-acetyl-AHBA was still the major metabolite, suggesting that NhoA is not solely responsible for AHBA acetylation. Additionally, NhoA may not have even been responsible for any acetylation. Next, alternative fermentation media were tested to see if one favored AHBA production. When Luria-Bertani (LB) medium was tested, AHBA and $N$-acetyl-AHBA were found at concentrations of $55 \mathrm{mg} /$ liter and $75 \mathrm{mg} /$ liter, respectively, after a 24 hours shake flask fermentation with BAPI. Lastly, a known inhibitor of NAT activity, glycyrrhizic acid [12], was added to an LB shake flask fermentation at a concentration of $10 \mathrm{mM}$. After 24 hours no $\mathrm{N}$-acetylAHBA was observed, and AHBA was found at $10 \mathrm{mg} / \mathrm{ml}$ in BAPI strains. Lower concentrations of glycyrrhizic acid failed to inhibit the acetylation reaction. While the use of glycyrrhizic acid yielded no $\mathrm{N}$-acetyl-AHBA side product formation, the use of this compound in ansamycin fermentations could prove to be impractical, as ansamycin cyclases are themselves NATs [13], and may be inhibited by glyccyrrhizic acid. If so, media formulations that leave out glucose, such as LB, would be the best alternative.

\section{Methoxymalonyl-ACP Production}

The five $f k b G-K$ genes from $S$. hygroscopicus are believed to be responsible for the production of methoxymalonylACP [9]. Previous work has suggested that an unknown glycolytic intermediate is first loaded onto the acyl carrier 
protein (ACP) FkbJ. This intermediate then presumably undergoes a series of oxidations and an $O$-methyl transfer to yield methoxymalonyl-ACP [14]. These five genes had been previously transferred to $S$. fradiae where midecamycin A was heterologously produced [15].

To determine if methoxymalonyl-ACP could be made in $E$. coli using the $f k b G-K$ operon, the five genes were first cloned into a pET28 expression vector. $F k b J$ was also cloned into a pET28 expression vector independently. Next, the $f k b$ operon was expressed in the E. coli strain BAP1 [2], which contains the $s f p$ phosphopantheniene transferase gene required for post-translational modification of ACPs. At least four of the five proteins were observed on a SDSPAGE gel after Ni-NTA column purification (Fig. 2). FkbH and FkbI have similar molecular masses, $39 \mathrm{kDa}$ and $38 \mathrm{kDa}$, respectively, and could not be resolved on a SDSPAGE gel after Ni-NTA purification. However, when subjected to HiTrap Q anion exchange separation, the two proteins eluted at different times (data not shown). If $E$. coli is able to synthesize the unknown precursor for methylmalonyl-thioester biosynthesis, it may be possible to detect methoxymalonyl-FkbJ. To determine if this was indeed the case, FkbJ was further purified from the other four Fkb proteins, as described in the Experimental Methods section. The protein was then digested with trypsin, and submitted for LC/MS analysis. The expected mass of the trypsin fragment containing the active site serine and the phosphopantheneine arm is $2785 \mathrm{~g} / \mathrm{mol}$. The expected mass of the same fragment with methoxymalonyl attached is $2900 \mathrm{~g} / \mathrm{mol}$. At 22.9 minutes two peaks were observed with masses of 1393.5 and $1404.5 \mathrm{~g} / \mathrm{mol}$, which

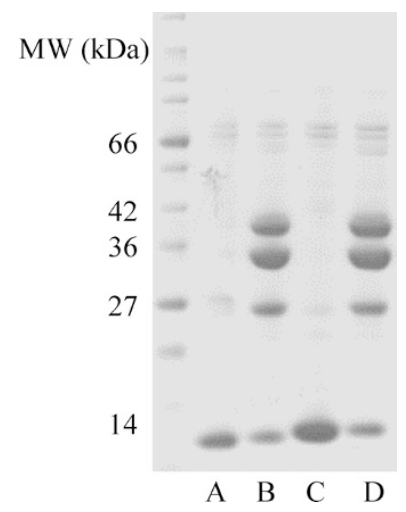

Fig. 2 Purified FkbH-K and GdmJ proteins after Ni-NTA purification.

Protein samples run on a polyacrylamide gel stained with Coomassie Brilliant Blue. Lane A, FkbJ expressed independently; Lane B, FkbG-K (24, 39, 38, 9.5, 29 kDa respectively) expressed simultaneously; lane C, GdmJ expressed independently; lane D, FkbG-I, -K, GdmJ (10 kDa) expressed simultaneously.
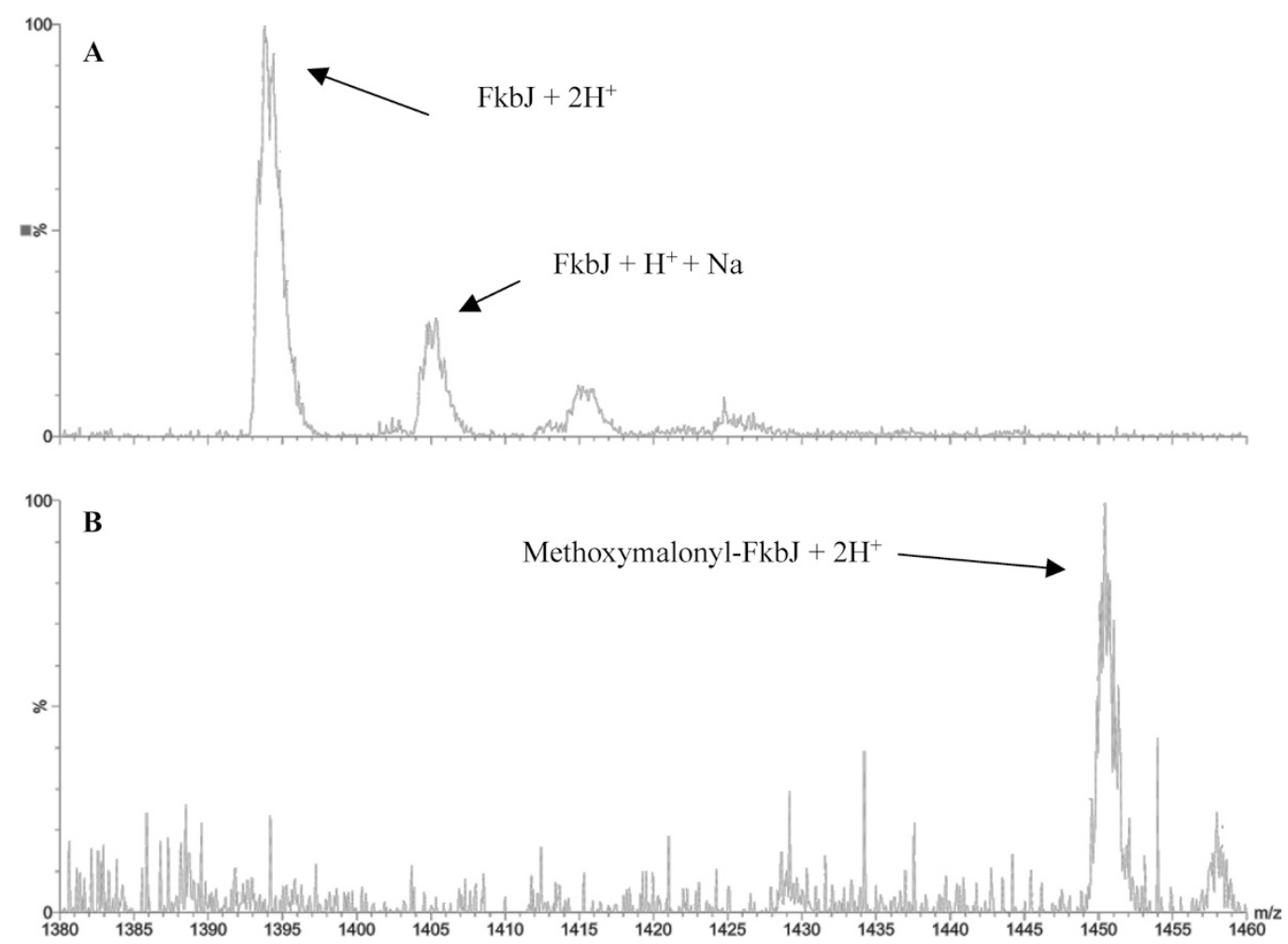

Fig. 3 LC/MS spectrum of FkbJ containing the phosphopantheniene arm.

(A) FkbJ expressed independently after digestion with trypsin. (B) FkbJ expressed with FkbG-I, -K after digested with trypsin. 
correspond to the $\mathrm{FkbJ}+2 \mathrm{H}^{+}$and $\mathrm{FkbJ}+\mathrm{H}^{+}+\mathrm{Na}^{+}$peaks, respectively (Fig. 3). At 18.5 minutes a peak was observed with a mass of $1451.4 \mathrm{~g} / \mathrm{mol}$ which corresponds to methoxymalonyl-FkbJ $+2 \mathrm{H}^{+}$. Based on the peak area ratios, approximately $20 \%$ of $\mathrm{FkbJ}$ was loaded with methoxymalonyl extender units. When FkbJ was expressed without the other Fkb proteins and subjected to similar analysis, only the $\mathrm{FkbJ}+2 \mathrm{H}^{+}$peak was observed. The methoxymalonyl-FkbJ $+2 \mathrm{H}^{+}$peak was absent.

The ability of FkbJ to interact with PKS modules from heterologous synthases has not been fully explored. Although it has been shown that FkbJ can productively interact with the midecamycin A polyketide proteins, it is unknown if FkbJ will interact with ansamycin polyketide proteins. For example, GdmJ, the homolog of FkbJ found in the geldanamycin pathway, has approximately 50\% sequence identity with FkbJ. This difference may prevent FkbJ from effectively transferring a methoxymalonyl extender unit to the geldanamycin PKS. To circumvent this potential problem, the corresponding methoxymalonyl-
ACP from the geldanamycin system, $G d m J$, was cloned with $F k b G H I K$ into the same pET28 expression vector. All five proteins were co-expressed (see Fig. 2) and GdmJ was purified, digested with chymotrypsin, and submitted for LC/MS analysis. The expected mass of the chymotrypsin fragment containing the active site serine and the phosphopantheneine arm is $1195 \mathrm{~g} / \mathrm{mol}$. The expected mass of the same fragment with methoxymalonyl attached is $1311 \mathrm{~g} / \mathrm{mol}$. At 13.6 minutes two peaks were observed with masses of 1196.3 and $1218.4 \mathrm{~g} / \mathrm{mol}$ that corresponds to the $\mathrm{GdmJ}+\mathrm{H}^{+}$and $\mathrm{GdmJ}+\mathrm{Na}^{+}$respectively. At 16.6 minutes a major peak was observed with mass of $1312.3 \mathrm{~g} / \mathrm{mol}$ that corresponds to methoxymalonyl-GdmJ $+\mathrm{H}^{+}$(Figs. 4 and 5). Approximately $60 \%$ of $\mathrm{GdmJ}$ was loaded with methoxymalonyl extender units, based on the peak area ratios. When GdmJ was expressed without the other Fkb proteins and subjected to the same analysis, only the $\mathrm{GdmJ}+\mathrm{H}^{+}$peak was observed. The methoxymalonyl$\mathrm{GdmJ} \mathrm{H}^{+}$peak was absent.

The ability to efficiently synthesize AHBA and
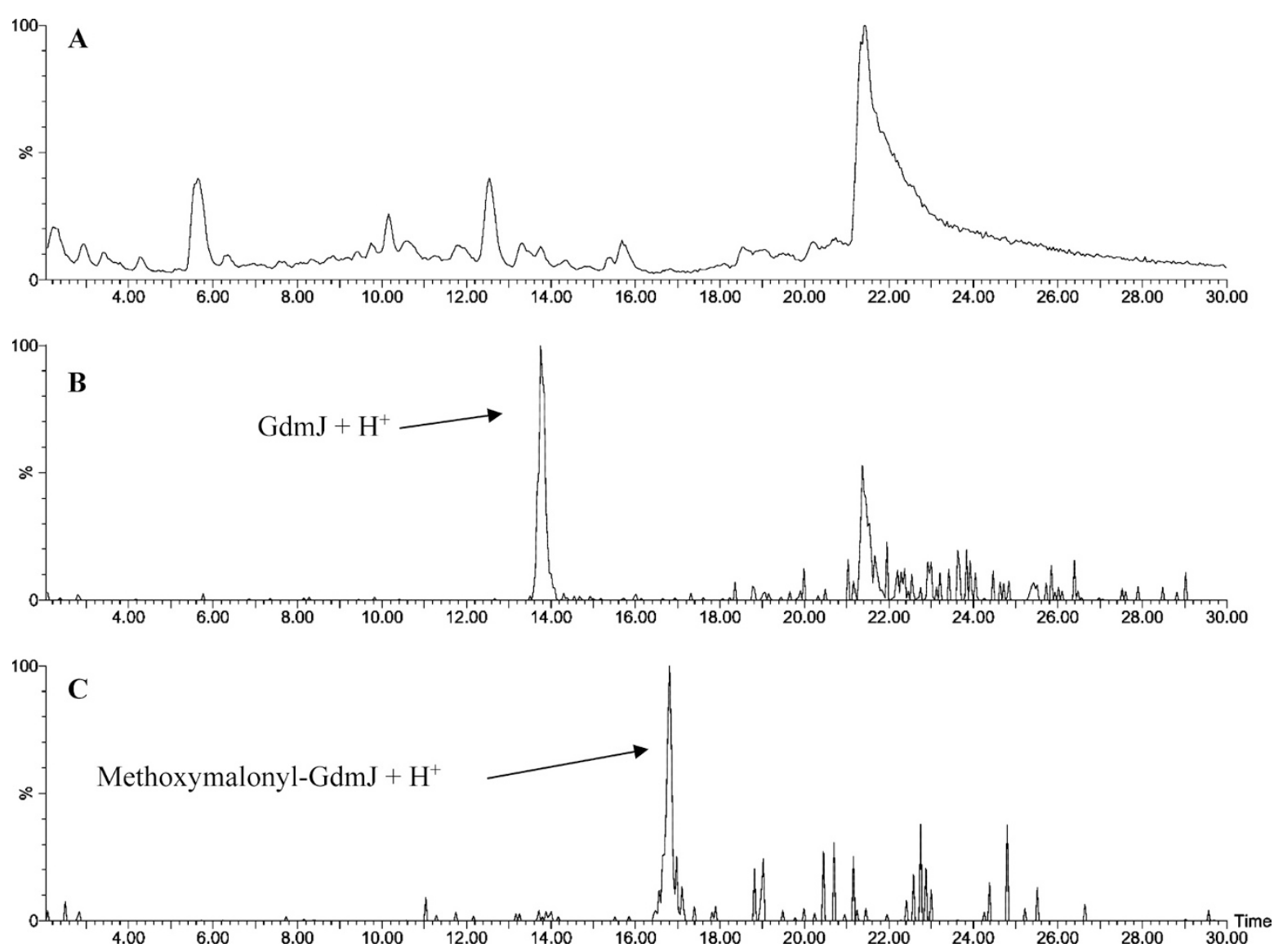

Fig. $4 \mathrm{LC} / \mathrm{MS}$ traces from 2 30 minutes for GdmJ digestion when co-expressed with FkbG-I, -K.

(A) Total ion count. Post 30 minutes, no new peaks were observed. (B) Extracted ion count for $1196 \mathrm{~g} / \mathrm{mol}$. (C) Extracted ion count for $1312 \mathrm{~g} / \mathrm{mol}$. 

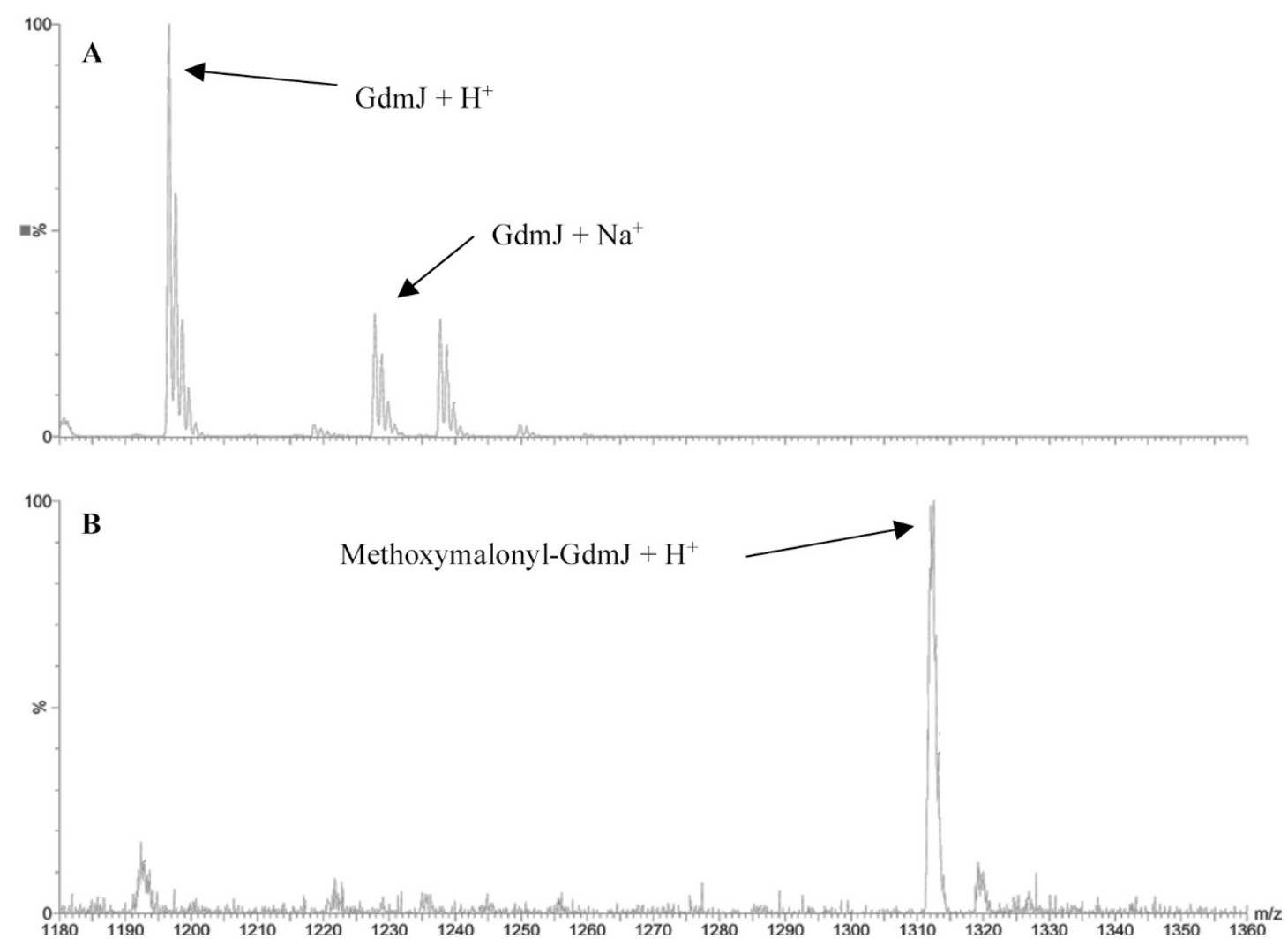

Fig. $5 \mathrm{LC} / \mathrm{MS}$ spectrum of GdmJ containing the phosphopantheniene arm.

(A) GdmJ expressed independently after digestion with chymotrypsin. (B) GdmJ expressed with FkbG-I, -K after digestion with chymotrypsin.

methoxymalonyl-ACP in E. coli expands the tools available to make polyketides in this bacterium. Previous work introduced necessary polyketide post-translational modifying enzymes and pathways to make the extender unit methylmalonyl-CoA in E. coli. The combination of these accomplishments should allow work to continue on expressing ansamycin polyketides in heterologous hosts.

\section{Experimental}

\section{AHBA Fermentations and Analysis}

pKW255, described previously, was used for all AHBA fermentations. pKW255 was first introduced via transformation into both BAP1 and MAR1 (see below for details). AHBA shake-flask fermentations were carried out by inoculating $100 \mathrm{ml}$ of either LB media or M9 minimal media with $0.5 \mathrm{ml}$ of the desired strain of $E$. coli from an overnight culture. Cultures were agitated at $37^{\circ} \mathrm{C}$ until an $\mathrm{OD}_{600}$ of 0.6 was reached. IPTG was added to a final concentration of $200 \mu \mathrm{M}$ and the culture was agitated at $30^{\circ} \mathrm{C}$ for 24 hours.
AHBA and $N$-acetyl-AHBA fermentation concentrations were determined by injecting $100 \mu$ l of clarified broth onto a Phenomenex C18 reverse phase HPLC column with a linear gradient between buffer A (water, $0.5 \%$ trifloroacetic acid) and buffer B (acetonitrile, $0.5 \%$ trifloroacetic acid) from $0 \%$ to $40 \%$ over 40 minutes at a flow rate of $1.0 \mathrm{ml} /$ minute. Authentic references were used to determine the retention time of the products and were also used to generate standard curves. AHBA was chemically synthesized as previously described [16]. $N$-Acetyl-AHBA was isolated from shake flask fermentations using methods previously described for isolating rifamycin intermediates [10]. $N$-Acetyl-AHBA; ${ }^{1} \mathrm{H}$ NMR (400 MHz, $\left.\mathrm{CD}_{3} \mathrm{OD}\right)$, $\delta=7.58(\mathrm{t}, J=1.6 \mathrm{~Hz}, 1 \mathrm{H}), \delta=7.42(\mathrm{t}, J=2.0 \mathrm{~Hz}, 1 \mathrm{H})$, $\delta=7.15(\mathrm{t}, J=1.6 \mathrm{~Hz}, 1 \mathrm{H}), \delta=2.10(\mathrm{~s}, 3 \mathrm{H}))$; MS (ESI+) calculated for $\mathrm{C}_{9} \mathrm{H}_{9} \mathrm{NO}_{4}\left[\mathrm{M}+\mathrm{Na}^{+}\right]$218.2, found 218.3 and MS (ESI-) calculated for $\left[\mathrm{M}-\mathrm{H}^{+}\right]$194.1, found 194.1.

\section{Gene Knockout}

To delete the putative E. coli NAT, nhoA, the temperature sensitive plasmid named pMAR46 was created as follows. First, regions approximately $1 \mathrm{~kb}$ upstream and downstream 
Table 1 Primer sequence used in PCR reactions

\begin{tabular}{|c|c|c|}
\hline $\begin{array}{c}\text { Gene or } \\
\text { chromosomal } \\
\text { region }\end{array}$ & Forward primer sequence $\left(5^{\prime} \sim 3^{\prime}\right)$ & Reverse primer sequence $\left(5^{\prime} \sim 3^{\prime}\right)$ \\
\hline Upstream of NhoA & GGTAAGCTTTACTGACATCTTTCAAAAGGAGCG & TATGGATCCGCATGCCAGGCGTTTCCCTTAGTGGTTTTC \\
\hline $\begin{array}{l}\text { Downstream } \\
\text { of NhoA }\end{array}$ & $\begin{array}{l}\text { GTAGGATCCATCGAATTTTATGTCAGGTTGCCGGA } \\
\text { TGCGG }\end{array}$ & TATGAGCTCGGAGAACGTTAATGAAACCGCAGG \\
\hline NhoA & GAAAACCACTAAGGGAAACGCCTG & \\
\hline$F k b G$ & GGTTTTCATATGGCTAATCAGATAACCCTGTCC & CTATGAATTC ACTAGTTCACCGTTTCCGCAGCAGGGT \\
\hline $\mathrm{FkbH}$ & GCATTTCATATGACCATTGTCAAGTGTCTGGTC & CATTGAATTC ACTAGTTCACCCTGCTGCGGAAACGGT \\
\hline \multirow[t]{2}{*}{ Fkbl } & GCATTTCATATGCCTGAGCGTGACGCGCTGCTG & GCTTTGAATTC \\
\hline & & ACTAGTTCAAGCCGGGAGCGCCAGCGC \\
\hline \multirow[t]{2}{*}{ FkbJ } & GCATTTCATATGACCGCACCCGTCAAGGACGAC & GCAAAGAATTC \\
\hline & & ACTAGTTCAGGCATCGACGGCCCGCAG \\
\hline$F k b K$ & GCATTTCATATGGACCACCAGCTGATCGTGCTC & GCATTGAATTC ACTAGTTCATGAGACACCTTCTCGTA \\
\hline GdmJ & TTTTCATATGACCATCTCATCCGAGCCGGGC & AAAAGAATTC ACTAGTTCACGGCTGTGCCCCGCGCAG \\
\hline
\end{tabular}

Engineered restriction sites are in bold.

of the NAT were amplified by PCR and ligated into PCRBlunt vectors. To the upstream fragment a HindIII site and a BamHI site were engineered. To the downstream fragment a BamHI site and a SacI site were added. Table 1 contains sequences of primers used. These fragments were assembled into pUC18 to form pNM4. Next, the cassette was transferred to pMAK705 [17] using HindIII and SacI, yielding pMAR46.

pMAR46 was introduced via transformation into the $E$. coli strain BAP1. Gene replacement via double crossover events was engineered as described elsewhere [17]. Transformants were screened with PCR using one primer designed $1 \mathrm{~kb}$ after the NAT and the second primer designed at the beginning of the NAT gene. Transformants containing the NAT gave a PCR product of $1.8 \mathrm{~kb}$, while transformants with the desired deletion yielded $1 \mathrm{~kb}$ PCR products.

\section{Construction of $f k b G-K$ and $f k b G-I,-K, g d m J$ Expression System}

To construct the fkb operons, PCR was first used to clone out each of the $f b k H-K$ genes as well as the $g d m J$ gene. An $N$-terminal $N d e I$ site and a $C$-terminal $E c o$ RI site followed by an SpeI site were engineered into each gene. Each gene was cloned into pET28b (Novagen) using NdeI and EcoRI. Table 1 contains the sequence of primers used. This generated pMAR113 and pMAR128 expression vectors for $f k b J$ and $g d m J$, respectively. To engineer the desired operons, XbaI-SpeI cassettes were assembled into a separate $\mathrm{pET} 28 \mathrm{~b}$ vector sequentially by taking advantage of compatible sticky ends produced by these two restriction enzymes. Each of the XbaI-SpeI cassettes included individual ribosome binding sites. This generated pMAR118 and pMAR130 expression vectors for $f k b G-K$ and $f k b G-I,-K, g d m J$ respectively.

\section{Expression and Purification of FkbG-K and FbkG-I, -K, GdmJ}

E. coli BAP1 [2] was used as a host to express the $f k b G H I J K$ and $g d m J$ genes. A single transformant was used to start $5 \mathrm{ml}$ of LB media cultures with kanamycin (50 mg/liter) at $37^{\circ} \mathrm{C}$ and $250 \mathrm{rpm}$. The starter culture was grown overnight and used to inoculate 1 liter of LB medium containing the same concentration of kanamycin. The culture was grown at $37^{\circ} \mathrm{C}$ and $250 \mathrm{rpm}$ until an OD of $0.6 \sim 0.8$ at which point the cultures were placed on ice for 15 minutes. Next the cultures were induced with $100 \mu \mathrm{M}$ of isopopropyl- $\beta$-D-thiogalactopyranoside and incubated at $18^{\circ} \mathrm{C}$ and $250 \mathrm{rpm}$ for 20 hours.

All purification procedures were performed on ice or at $4^{\circ} \mathrm{C}$. FkbJ and GdmJ were isolated by first spinning the cell culture at $2,500 \times g$ for 20 minutes and resuspending the cell pellet in disruption buffer $(200 \mathrm{mM}$ sodium phosphate, pH 7.2/200 mM sodium chloride/0.2 mM DTT/1.5 mM benzamine $/ 2 \mathrm{mg} /$ liter pepstatin $/ 2 \mathrm{mg} /$ liter leupeptin $/ 30 \%$ glycerol). Resuspended cells were lysed using sonication and then clarified at $40,000 \times g$ for 60 minutes. The lysate was then equilibrated with $2 \mathrm{ml}$ of Ni-NTA resin (Qiagen) for 1 hour. The resin was first rinsed with $10 \mathrm{mM}$ imidazole in a pH $7.2100 \mathrm{mM}$ sodium phosphate buffer, and then the protein was eluted off the resin with $200 \mathrm{mM}$ imidazole in the same buffer. Additional purification was carried out 
using anion-exchange chromatography. The protein solution was loaded onto a HiTrap Q $5 \mathrm{ml}$ column (Amersham). A gradient of $0 \sim 1 \mathrm{M}$ sodium chloride in $100 \mathrm{mM}$ HEPES (pH 7.2), $2.0 \mathrm{mM}$ DTT, and $10 \% \mathrm{vol}$ glycerol was run at $3 \mathrm{ml} /$ minute for 15 column volumes. Three $\mathrm{ml}$ fractions were collected, and those fractions containing FkbJ or GdmJ were pooled, concentrated, and buffer exchanged into $100 \mathrm{mM}$ sodium phosphate ( $\mathrm{pH} 7.2$ ), $2.0 \mathrm{mM}$ DTT, 20\% vol glycerol using an Amcion Ultra protein concentrator (Waters). Purified protein was analyzed on a $4 \sim 12 \%$ Bis-Tris polyacrylamide gel (Invitrogen) stained with Coomassie brilliant blue stain. This protein purification procedure yielded $10 \mathrm{mg} /$ liter of purified FkbJ or GdmJ.

\section{LC/MS Analysis of FkbJ and GdmJ}

FkbJ was digested with trypsin and GdmJ was digested with chymotrypsin as follows. Each digestion was carried out in $50 \mu \mathrm{l}$ reaction with $100 \mathrm{mM}$ sodium phosphate $(\mathrm{pH}$ 7.2), $100 \mu \mathrm{M} \mathrm{FkbJ}$ or GdmJ, and $2.5 \mu \mathrm{M}$ trypsin or chymostrypsin. The reactions were incubated at $30^{\circ} \mathrm{C}$ for 60 minutes. The reactions were then quenched with $50 \mu \mathrm{l}$ of a $10 \%$ formic acid solution.

Fifty microliters of each quenched reaction were analyzed using a Surveyor HPLC system (ThermoFinnigan) equipped with a Vydac $\mathrm{C} 18$ reverse phase polymer column. Mass spectra were collected on an LCQ quadrupole ion trap (ThermoFinnigan) mass spectrometer equipped with an electrospray ion source operating in positive ion mode. The sheath gas was set to 60 (arbitrary units), spray voltage to $4.5 \mathrm{kV}$, and capillary temperature to $200^{\circ} \mathrm{C}$. A linear gradient between buffer A (water, $0.1 \%$ formic acid) and buffer B (acetonitrile, $0.1 \%$ formic acid) from $5 \%$ to $95 \%$ over 60 minutes at a flow rate of $0.2 \mathrm{ml} /$ minute was used.

Acknowledgment This research was supported by a grant from the NIH (AI38947 to C.K). M.A.R is a recipient of a Stanford Graduate Fellowship. We thank the Vincent Coates Stanford University MS facility for help with mass spectrometric analysis.

\section{References}

1. Rude MA, Khosla C. Engineered biosynthesis of polyketides in heterologous hosts. Chem Eng Sci 59: 4693-4701 (2004)

2. Pfeifer BA, Admiraal SJ, Gramajo H, Cane DE, Khosla C. Biosynthesis of complex polyketides in a metabolically engineered strain of E-coli. Science 291: 1790-1792 (2001)

3. Prelog V, Oppolzer W. Ansamycins, new class of microbial metabolites. Helv Chim Acta 56: 2279-2287 (1973)

4. Stratmann A, Toupet C, Schilling W, Traber R, Oberer L,
Schupp T. Intermediates of rifamycin polyketide synthase produced by an Amycolatopsis mediterranei mutant with inactivated rifF gene. Microbiol-Uk 145: 3365-3375 (1999)

5. Floss HG, Yu TW. Lessons from the rifamycin biosynthetic gene cluster. Curr Opin Chem Biol 3: 592-597 (1999)

6. August PR, Tang L, Yoon YJ, Ning S, Mèuller R, Yu TW, Taylor M, Hoffmann D, Kim CG, Zhang X, Hutchinson CR, Floss HG. Biosynthesis of the ansamycin antibiotic rifamycin: deductions from the molecular analysis of the rif biosynthetic gene cluster of Amycolatopsis mediterranei S699. Chem Biol 5: 69-79 (1998)

7. Rascher A, Hu Z, Viswanathan N, Schirmer A, Reid R, Nierman WC, Lewis M, Hutchinson CR. Cloning and characterization of a gene cluster for geldanamycin production in Streptomyces hygroscopicus NRRL 3602. FEMS Microbiol Lett 218: 223-230 (2003)

8. Yu TW, Bai L, Clade D, Hoffmann D, Toelzer S, Trinh KQ, $\mathrm{Xu}$ J, Moss SJ, Leistner E, Floss HG. The biosynthetic gene cluster of the maytansinoid antitumor agent ansamitocin from Actinosynnema pretiosum. Proc Natl Acad Sci USA 99: 7968-7973 (2002)

9. Carroll BJ, Moss SJ, Bai L, Kato Y, Toelzer S, Yu TW, Floss HG. Identification of a set of genes involved in the formation of the substrate for the incorporation of the unusual "glycolate" chain extension unit in ansamitocin biosynthesis. J Am Chem Soc 124: 4176-4177 (2002)

10. Watanabe K, Rude MA, Walsh CT, Khosla C. Engineered biosynthesis of an ansamycin polyketide precursor in Escherichia coli. Proc Natl Acad Sci USA 100: 9774-9778 (2003)

11. Yamamura E, Sayama M, Kakikawa M, Mori M, Taketo A, Kodaira K. Purification and biochemical properties of an $\mathrm{N}$ hydroxyarylamine $O$-acetyltransferase from Escherichia coli. Biochim Biophys Acta 1475: 10-16 (2000)

12. Lo HH, Yen YS, Hsieh SE, Chung JG. Glycyrrhizic acid inhibits arylamine $N$-acetyltransferase activity in Klebsiella pneumoniae in vitro. J Appl Toxicol 17: 385-390 (1997)

13. Pompeo F, Mushtaq A, Sim E. Expression and purification of the rifamycin amide synthase, RifF, an enzyme homologous to the prokaryotic arylamine $\mathrm{N}$ acetyltransferases. Prot Expr Purif 24: 138-151 (2002)

14. Watanabe K, Khosla C, Stroud RM, Tsai SC. Crystal structure of an Acyl-ACP dehydrogenase from the FK520 polyketide biosynthetic pathway: insights into extender unit biosynthesis. J Mol Biol 334: 435-444 (2003)

15. Rodriguez E, Ward S, Fu H, Revill WP, McDaniel R, Katz L. Engineered biosynthesis of 16-membered macrolides that require methoxymalonyl-ACP precursors in Streptomyces fradiae. Appl Microbiol Biotechnol 66: 85-91 (2004)

16. Hartung IV, Rude MA, Schnarr NA, Hunziker D, Khosla C. Stereochemical assignment of intermediates in the rifamycin biosynthetic pathway by precursor-directed biosynthesis. J Am Chem Soc 127: 11202-11203 (2005)

17. Hamilton CM, Aldea M, Washburn BK, Babitzke P, Kushner SR. New method for generating deletions and gene replacements in Escherichia coli. J Bacteriol 171: 4617-4622 (1989) 